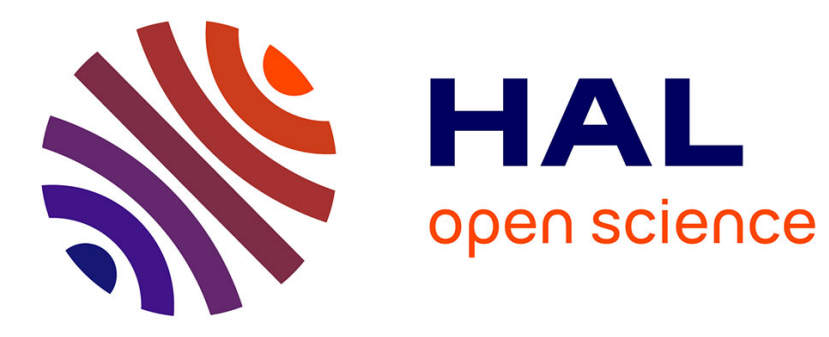

\title{
Le rôle des psychologues dans le contexte de la sécurité routière
}

\author{
Béatrice Degraeve, Catherine Gabaude, Sébastien Laurent, Laurence \\ Paire-Ficout
}

\section{- To cite this version:}

Béatrice Degraeve, Catherine Gabaude, Sébastien Laurent, Laurence Paire-Ficout. Le rôle des psychologues dans le contexte de la sécurité routière. Le Journal des psychologues, 2018, 2018 (360), pp. 14-18. 10.3917/jdp.360.0014 . hal-01972365

\section{HAL Id: hal-01972365 https://hal.science/hal-01972365}

Submitted on 7 Jan 2019

HAL is a multi-disciplinary open access archive for the deposit and dissemination of scientific research documents, whether they are published or not. The documents may come from teaching and research institutions in France or abroad, or from public or private research centers.
L'archive ouverte pluridisciplinaire HAL, est destinée au dépôt et à la diffusion de documents scientifiques de niveau recherche, publiés ou non, émanant des établissements d'enseignement et de recherche français ou étrangers, des laboratoires publics ou privés. 
Merci de citer cet article avec les références suivantes :

Degraeve, B., Gabaude, C., Laurent, S., Paire-Ficout, L. (2018). Le rôle des psychologues dans le contexte de la sécurité routière. Le journal des psychologues 2018/8 n³60, 14-18.

\section{Béatrice Degraeve}

Docteur en psychologie sociale

Ingénieur recherche IFSTTAR, TS2-LESCOT

\section{Catherine Gabaude}

Chargée de recherche en neurosciences et psychologie

IFSTTAR, TS2-LESCOT

\section{Sébastien Laurent}

Doctorant en psychologie cognitive

IFSTTAR, TS2-LESCOT

\section{Laurence Paire-Ficout}

Chargée de recherche en psychologie cognitive

IFSTTAR, TS2-LESCOT

Le rôle des psychologues dans le contexte de la sécurité routière : formateurs, évaluateurs, rééducateurs et acteurs de la recherche

Malgré les apports indéniables de la psychologie pour promouvoir la sécurité routière en France, les places qu'occupent les psychologues dans les dispositifs de formation, d'évaluation et de réhabilitation des usagers de l'espace routier ne sont pas toujours bien identifiées, aussi bien au niveau de leurs pratiques de terrain que de leurs contributions aux actions de recherche. En effet, ces dernières années, les travaux menés dans le domaine de la psychologie du transport se sont concentrés sur le rôle des perceptions, des motivations morales, des valeurs, des normes personnelles, des croyances et des attitudes, des intentions personnelles et des habitudes pour comprendre les comportements adoptés par les usagers des transports. De nombreux déterminants des consommations de la mobilité ont été pris en compte, mais ces dimensions ne sont pas 
suffisantes pour aborder les questions en lien avec la problématique de l'aptitude à la conduite, appelant à l'étude des troubles mentaux et des symptômes de troubles mentaux comme variables affectant les comportements des usagers (Gössling, 2013).

À travers cet article, nous cherchons à dresser un panorama des secteurs dans lesquels les psychologues interviennent dans ce domaine particulier de la psychologie clinique du transport. À partir des recherches menées par les psychologues et de leurs actions menées sur le terrain, nous souhaitons montrer les apports de la psychologie dans l'amélioration du dispositif d'évaluation et de suivi de l'aptitude des conducteurs. Nous chercherons plus particulièrement à souligner ces apports dans la formation à la sécurité routière, l'évaluation de l'aptitude à la conduite, mais également, plus en amont, dès l'accès au permis de conduire et en particulier l'obtention du code de la route. Une meilleure coordination (à l'intérieur des communautés, mais aussi entre les communautés d'acteurs en développant une approche pluriprofessionnelle) semble nécessaire pour apporter une réponse adaptée à ces problématiques qui sont à l'interface des politiques de santé publique et de sécurité routière.

\section{LE RÔLE DES PSYCHOLOGUES ET LA PROBLÉMATIQUE DU PERMIS DE CONDUIRE}

\section{Les apports dans le cadre de la formation à la sécurité routière}

Les psychologues ont une connaissance des processus et des mécanismes cognitifs et psychosociaux qui peuvent influencer le comportement individuel. L'appréhension des effets de certains traits de personnalité sur les comportements, notamment à risques ${ }^{1}$, ainsi que des processus cognitifs ou émotionnels leur permettent de comprendre et de répondre à la problématique de la transgression des règles et de la mise en danger de soi sur la route. Ces compétences peuvent être mises au service de l'accompagnement et de la réhabilitation, le psychologue ayant les clés pour guider les changements d'attitude et de comportement $-\mathrm{y}$ compris lorsque certaines problématiques spécifiques entrent également en jeu (addictions, troubles du comportement, anxiété, dépression, vieillissement pathologique....). Ils trouvent donc naturellement leur place dans la formation à la sécurité routière, notamment à travers les stages de sensibilisation à la sécurité routière à destination des conducteurs infractionnistes. Leur travail consiste à sensibiliser les stagiaires aux enjeux de la sécurité routière et à susciter l'émergence de nouveaux comportements plus sûrs pour soi et pour autrui (Nallet-Buisson, Makhlouf, 2010).

Concernant la formation à la sécurité routière effectuée au niveau préventif, on constate cependant que les psychologues sont rarement associés à ces actions, essentiellement prises en charge par d'autres acteurs (gendarmerie, police nationale, pompiers, bénévoles...). Pourtant, des travaux montrent que les actions de prévention menées dans les lycées français entre 2012 et 2014 ont 
permis aux élèves d'augmenter leurs connaissances du risque et de mieux appréhender les dangers de la route ${ }^{2}$. Compte tenu des enjeux en termes de santé publique et des compétences que les psychologues de terrain ont à offrir dans ce domaine, on ne peut que regretter qu'ils ne soient pas davantage associés à ces actions de sensibilisation préventives et d'éducation à la sécurité routière. Leur implication devrait être engagée plus en amont, en concertation avec les différents acteurs concernés, plutôt qu'uniquement au moment de l'infraction, après la mise en danger de soi et d'autrui. Leurs capacités de détecter et d'analyser les mécanismes individuels et sociaux soustendant le raisonnement et le comportement, ainsi que leurs capacités d'induire un changement malgré les potentielles résistances individuelles, permettraient d'apporter une plus-value importante à l'action collective.

\section{Leur rôle dans l'examen psychotechnique prévu dans le cadre du contrôle médical de l'aptitude à}

\section{la conduite}

En cas d'annulation (retrait immédiat du permis), d'invalidation (perte progressive de tous les points du permis) ou en cas de suspension du permis de conduire pour une durée égale ou supérieure à six mois, une visite médicale et un examen psychotechnique sont obligatoires pour le conducteur (décret n² 2016-39 du 22 janvier $2016^{3}$; l'arrêté du 26 août $2016^{4}$ ). Ces situations recouvrent des motifs différents et des profils variés de conducteurs (ceux qui adoptent des comportements délictueux ou qui présentent un état de santé dégradé). Le permis peut être retiré ou suspendu à la suite d'une infraction grave (conduite en état d'alcoolémie avancée ou sous l'emprise de stupéfiant, dépassement de $30 \mathrm{~km}$ / h ou plus de la vitesse autorisée, délit de fuite, refus d'obtempérer...) ou d'infractions moins sévères mais répétées et sur une période restreinte menant à la perte de tous les points (dépassement dangereux, circulation en sens interdit, franchissement d'une ligne continue, non-respect d'un panneau stop...). Le profil des conducteurs qui commettent ces infractions est également très varié : il peut s'agir d'un conducteur présentant une addiction, une opposition aux règles ou à l'autorité, une tendance à la prise de risque, une défaillance cognitive (défaut d'attention, perturbation de la mémoire, du traitement de l'information et son actualisation, de la prise de décision...) ou encore une maladie ou une pathologie impactant la conduite (lésions cérébrales, maladies chroniques, maladies psychiatriques...).

Ici, le rôle du psychologue est donc de procéder à l'évaluation psychologique et psychotechnique du conducteur, par le biais d'un entretien individuel et la passation de tests permettant d'appréhender ses capacités psychomotrices, sensorielles ou cognitives. Ces éléments sont consignés dans un compte rendu d'examen à destination du médecin agréé par la préfecture (ou de la commission médicale préfectorale suivant le type d'infraction(s) commise(s) par le candidat). Le psychologue émet donc un avis favorable (avec ou sans restriction) ou un avis défavorable. Le médecin agréé 
intègre ensuite les éléments qui lui sont parvenus pour formuler un avis complet et le transmettre à la préfecture pour qu'une décision d'aptitude ou d'inaptitude soit prise et qu'elle soit délivrée au conducteur et ainsi l'autoriser ou non à reprendre la conduite.

L'arrêté du 26 août 2016 (rédigé en concertation avec des psychologues) formule des préconisations concernant le choix des tests psychotechniques et la réalisation de l'entretien individuel requis dans le cadre du contrôle médical de l'aptitude à la conduite. Cet arrêté insiste également sur la complémentarité entre la phase d'entretien et la phase de passation des tests. En effet, l'entretien permet de discerner les capacités psychomotrices, sensorielles ou cognitives devant faire l'objet d'une évaluation (il aide à orienter le choix des tests), mais permet également de prendre en compte certains éléments déterminants qui dépassent le cadre spécifique de ces tests (tels que les abus ou la dépendance à des substances, les problématiques psychiatriques, certains comportements socialement inadaptés). Ces deux composantes de l'examen psychotechnique (entretien et tests) donnent donc des informations distinctes, qui sont toutes les deux indispensables pour évaluer les risques routiers auxquels le conducteur s'expose et expose les autres.

Toutefois, si les psychologues ont su investir ce domaine et se saisir de ses enjeux, ils ne se sont pas encore constitués en une communauté structurée et fédérée (ce qui, par conséquent, induit un manque de visibilité aux niveaux national et international). Les différents acteurs de ce champ d'intervention (psychologues, chercheurs, médecins) ont de rares opportunités de se rencontrer et d'échanger pour enrichir et améliorer la prise en charge des conducteurs à risque, ce qui n'incite pas à une évolution souhaitable des modalités d'évaluation et de réhabilitation des conducteurs. Il est nécessaire de mettre en place une meilleure coordination (à l'intérieur des communautés, mais aussi entre les communautés d'acteurs en développant une approche pluriprofessionnelle) pour apporter une réponse adaptée à cette problématique de santé publique.

\section{LA RECHERCHE EN PSYCHOLOGIE POUR AMÉLIORER L'ÉVALUATION ET L'ACCOMPAGNEMENT DES CONDUCTEURS}

\section{L'aptitude à la conduite automobile : une activité complexe à évaluer}

Le code de la sécurité routière en France adopte un principe général fort qui vise à responsabiliser le conducteur : tout candidat au permis de conduire qui rencontre un problème de santé doit, de sa propre initiative, se soumettre à un contrôle médical ; il en est de même pour le conducteur atteint d'une affection médicale risquant de compromettre la sécurité routière ; cette démarche est également obligatoire pour obtenir un aménagement du droit de conduire ${ }^{5}$. Si une réglementation complexe existe en matière d'évaluation d'aptitude à la conduite ${ }^{6}$, elle ne décrit pas les moyens et modalités d'évaluation pour apporter une réponse adaptée à cette double 
problématique de santé publique et de sécurité routière. Les travaux de recherche menés sur cette problématique attestent de la difficulté à évaluer l'aptitude à la conduite automobile tant cette activité de la vie quotidienne est complexe, et les régulations comportementales mises en œuvre par les conducteurs et les autres usagers de la route sont nombreuses.

Les capacités motrices, perceptives et cognitives du conducteur sont sollicitées, de même que ses capacités métacognitives (sentiment de bien conduire, auto-évaluation de ses propres capacités de gérer des situations de conduite complexes voire critiques...) et sociocognitives (anticipation du comportement d'autrui et ajustement dynamique au comportement d'autrui...), ses états motivationnels (gestion des buts poursuivis...) et émotionnels (gestion de la colère, l'agressivité) ; la connaissance de ses propres mécanismes physiologiques est aussi requise (gestion de la fatigue, de la somnolence, de l'anxiété...). Au regard de ces éléments, il semble évident que la place du psychologue dans le processus d'évaluation des aptitudes à la conduite est déterminante.

\section{De la détection à la réhabilitation : vers une approche pluriprofessionnelle}

En France, différents acteurs sont aujourd'hui impliqués dans le repérage des conducteurs susceptibles d'être confrontés à des limitations de leurs aptitudes à la conduite. Les médecins généralistes et-ou les spécialistes peuvent, lors des phases d'anamnèse, déceler les faisceaux d'indices interrogeant l'aptitude à la conduite de leur patient. Ils peuvent ainsi leur prodiguer des conseils pour inciter leurs patients à adapter leurs comportements et modifier leurs habitudes de conduite, voire de mobilité. Les professionnels de santé travaillant auprès des médecins dans des établissements de soins de suite et de réadaptation (SSR : ergothérapeutes, neuropsychologues et psychologues) peuvent aussi apporter des informations importantes quant au suivi de leurs patients. Les forces de l'ordre (police ou gendarmerie), en verbalisant les conducteurs adoptant des comportements délictueux, contribuent aussi au repérage des conducteurs pouvant présenter une inaptitude à la conduite puisqu'après une suspension supérieure à un mois, une invalidation ou une annulation du permis de conduire, une évaluation de leur aptitude médicale à la conduite est requise.

Deux systèmes d'évaluation de l'aptitude à la conduite existent en France, mais sule-seul le premier système est habilité à prendre une décision en matière d'aptitude ou d'inaptitude à la conduite : - dans le cadre du suivi administratif du permis de conduire, les visites peuvent être réalisées auprès des médecins agréés pour le permis de conduire ou des commissions médicales préfectorales; - dans le cadre d'un programme de réadaptation ou de rééducation fonctionnelle, des équipes pluriprofessionnelles prennent en charge un patient, afin de faciliter son insertion. Elles sont très souvent confrontées à cette problématique de l'évaluation des capacités de conduite. 
La Haute autorité de santé a très récemment publié des recommandations de bonnes pratiques afin d'aider les praticiens et leur patient à aller vers une décision d'aptitude à la conduite la plus juste possible ${ }^{7}$. En l'absence de preuves scientifiques établies, ces recommandations sont pour l'instant fondées sur un accord d'experts. II reste donc de nombreux travaux de recherche à mener pour asseoir ces recommandations. Néanmoins, la conclusion unanime sur la nécessité de réaliser une évaluation pluriprofessionnelle sur route, afin de pouvoir émettre une décision d'aptitude à la conduite est une avancée majeure dans la prise en charge et le suivi des patients. Toutefois, en raison du respect du secret médical, les bilans d'évaluation produits ne peuvent être directement transmis à un médecin agréé. Des avis et décisions contradictoires peuvent ainsi être rendus aux patients. Le système de suivi des conducteurs reste à ce jour perfectible.

En l'absence de statistiques officielles, il est à ce jour difficile d'estimer le nombre de personnes évaluées annuellement à travers les deux dispositifs décrits précédemment, mais ce chiffre pourrait atteindre les 500000 (dont 300000 victimes de lésion cérébrale non évolutive). Ces évaluations représentent donc, pour les psychologues réalisant les examens psychotechniques et les neuropsychologues, un travail considérable, d'autant plus si l'on prend en compte la place du processus rééducatif après l'évaluation. L'intégration sociale des personnes présentant un trouble de la personnalité ou un handicap interroge à la fois les politiques de santé publique et de sécurité routière. Il convient de définir le meilleur processus de repérage, d'évaluation et d'accompagnement des patients pour lesquels la question de la reprise de la conduite automobile se pose. À ce jour, l'accompagnement consiste principalement à délivrer des informations sur les démarches médicoadministratives et financières pour le retour à la conduite. II reste des progrès à réaliser, afin de développer des procédures de rééducation et de réentraînement à la conduite, mais aussi de formation à l'usage des aides techniques qui, avec l'avènement des véhicules automatisés, voire autonomes, devraient pouvoir offrir des solutions pour le maintien de la mobilité en cas de contreindication médicale à la conduite d'un véhicule ordinaire. En tout état de cause, la place des psychologues dans l'amélioration de ces procédures est essentielle.

\section{LA RECHERCHE EN PSYCHOLOGIE POUR IDENTIFIER ET RÉPONDRE AUX DIFFICULTÉS D’ACCÈS AU CODE DE LA ROUTE}

\section{La question de l'accès au permis pour tous}

Au-delà des questions liées à l'évaluation de l'aptitude et à la réhabilitation des conducteurs, le rôle de la psychologie se pose également en amont, dès que se pose la question de l'accès au permis de conduire et, en particulier, la nécessité d’obtenir le code de la route. 
L'apprentissage des règles de sécurité routière démarre dès l'école primaire, mais elles ne sont appréhendées et intégrées dans leur ensemble par les apprentis conducteurs que lors de l'apprentissage du code de la route. L'examen du code de la route comporte une série de quarante questions à choix multiples et n'est réussi que si le candidat a répondu correctement à trente-cinq d'entre elles. Chaque question est illustrée par une photo et, depuis la réforme du code de la route de 2016, quatre questions sont accompagnées d'une vidéo.

Pour réussir, le candidat doit se livrer à un apprentissage et à un entraînement soutenu afin d'acquérir toutes les connaissances techniques et les fondements des règles. Au fur et à mesure de son apprentissage, le candidat réussit à déjouer les pièges de certaines questions en aiguisant son habileté à analyser rapidement et en profondeur tous les éléments de la scène.

Mais l'exercice est particulièrement périlleux pour des candidats à profils spécifiques, tels que les jeunes ayant des difficultés d'apprentissage ou des difficultés dans le traitement du langage écrit. C'est le cas des personnes dyslexiques et dysphasiques (" dys »), mais aussi des personnes sourdes qui présentent un retard à l'écrit majeur. Les candidats dyspraxiques ou cérébrolésés (à la suite d'un traumatisme crânien) peuvent également se retrouver en échec. Selon un rapport parlementaire de 2005 , le taux de réussite à l'examen du code de la route était de $58 \%$ pour les candidats francophones, alors qu'il était de $27 \%$ pour les candidats ne maîtrisant pas la langue française ${ }^{8}$. Malgré l'instauration d'aménagements pour ces candidats (" dys ", non-francophones, sourds, cérébrolésés) ${ }^{9}$, les taux de réussites restent faibles (Paire-Ficout, Borgetto, 2012).

\section{L'apport de la psychologie pour faciliter l'obtention du code de la route}

Actuellement, le matériel d'apprentissage du code de la route (manuels d'apprentissage, séries de questions à choix multiples, sites Internet) repose essentiellement sur l'écrit. Au-delà du fait que ce matériel peut représenter un obstacle pour certains candidats, son caractère statique ne facilite pas l'immersion dans l'activité de conduite et ne permet pas de transmettre les relations tempsdistances-vitesse.

Par rapport à ces questions, un des enjeux de la recherche en psychologie a consisté à développer et à tester un nouveau type de matériel pour examiner ces effets sur l'apprentissage et l'accès au permis. Les premiers résultats de travaux menés dans cette direction ont permis de montrer qu'un matériel pédagogique animé et conçu de telle façon que le candidat voit la scène comme s'il était placé derrière le volant du véhicule (plan subjectif) permet d'améliorer significativement les prises de décision dans un contexte de scène routière. Les candidats se montrent plus performants pour décider s'il est possible ou non de doubler ou de s'insérer dans une intersection ou sur l'autoroute quand la scène est animée que lorsqu'elle est statique ${ }^{10}$. 
Or, plusieurs animations conçues dans le cadre de la réforme du code de la route de 2016 ne respectent pas ces principes de base. Par exemple, il est préférable d'éviter un point de vue en aérien (moins immersif), une vitesse de défilement de l'animation trop rapide, d'animer une situation quand ce n'est pas nécessaire. Ce constat milite en faveur d'un rapprochement entre les travaux issus de la recherche et les éditeurs de tests qui conçoivent le matériel pédagogique du code de la route dans l'optique d'obtenir un matériel plus adapté aux apprenants, mais également plus cohérent envers un objectif d'apprentissage procédural de l'activité de conduite.

\section{CONCLUSION}

Si les apports de la psychologie sont indéniables pour promouvoir la sécurité routière, et ce, à travers un éventail d'actions variées, la communauté d'acteurs (psychologues, chercheurs, médecins) manque de fédération, de concertation et de coordination. Ce travail permet de dégager de nombreuses voies d'amélioration pour la détection, la prise en charge et l'accompagnement des conducteurs, tant ceux ayant des comportements à risque que ceux ayant connu un accident de la vie compromettant le retour à la conduite.

Au regard des avancées scientifiques récentes (recommandations HAS), il convient d'accompagner et de systématiser la mise en place d'une évaluation pluriprofessionnelle de l'aptitude à la conduite sur l'ensemble du territoire. Une meilleure concertation des acteurs impliqués dans les deux systèmes d'évaluation de l'aptitude à la conduite cohabitant aujourd'hui (médecins agréés et professionnels de santé SSR) permettrait des avancées importantes au niveau de la prise en charge et de l'accompagnement des patients. En matière de recherche, il paraît également nécessaire de réunir les éléments de preuve scientifique qui permettront de dépasser les accords d'experts actuels et de tendre vers des recommandations plus robustes. Enfin, il conviendrait de réfléchir à l'amélioration des dispositifs de formation existants pour les professionnels concernés par les questions de l'aptitude à la conduite (et, plus largement, de sécurité routière). Si les formations continues semblent nécessaires pour mettre à jour les connaissances, un élargissement de l'offre de formations initiales dédiées à ces questions pourrait être utile pour former une série de professionnels à ces enjeux spécifiques, voire susciter des vocations. Compte tenu de l'importance des enjeux attachés à ces questions, les réflexions et les actions à mener autour des bonnes pratiques méritent d'être investies par l'action collective.

\section{Notes}


1. Comme la recherche de sensations ou l'impulsivité (Zuckerman, 1979 ; Harden, Tucker-Drob, 2011).

2. Kraïem S. et al., 2014, Projet Prévention et éducation routières en lycée : évaluation des actions (PERLE). Rapport Final, Convention IFSTTAR-DSCR n 2200626575.

3. https://www.legifrance.gouv.fr/eli/decret/2016/1/22/INTS1510984D/jo

4. https://www.legifrance.gouv.fr/eli/arrete/2016/8/26/INTS1621322A/jo/texte

5. https://www.service-public.fr/particuliers/vosdroits/F2686

6. De nombreux textes révisés sont disponibles en consultant les deux décrets principaux : contrôle médical de l'aptitude à la conduite et diverses dispositions en sécurité routière ; et les quatre arrêtés : établissement et délivrance du permis de conduire, liste des affections médicales incompatibles avec l'obtention ou le maintien du permis de conduire ou pouvant donner lieu à la délivrance de permis de conduire de durée de validité limitée, organisation du contrôle médical, montant des honoraires des médecins agréés.

7. Voir les recommandations de la HAS, 2016, https://www.has-

sante.fr/portail/jcms/c_2590287/fr/label-de-la-has-reprise-de-la-conduite-automobile-apres-lesioncerebrale-acquise-non-evolutive.

8. Bertrand J. M., 2005, Faciliter l'accès des jeunes au permis de conduire : étude et propositions, Rapport parlementaire.

9. La loi sur le handicap de 2005 préconise l'instauration d'examens dédiés, d'un tiers-temps supplémentaire et la présence d'un interprète pendant l'examen en cas de besoin.

10. Laurent S., Paire-Ficout L., Argon S., Boucheix J.-M., 2017, « L'animation 3D comme support au code de la route. Intérêt pour les candidats sourds ", $9^{e}$ Conférence de psychologie ergonomique : ergonomie des technologies pour le développement des compétences, du 5 au 7 juillet 2017, à Dijon.

\section{Bibliographie}

Gössling S., 2013, « Urban Transport Transitions : Copenhagen, City of Cyclists », Journal of Transport Geography, 33 : 196-206.

Harden K. P., Tucker-Drob E. M., 2011, « Individual Differences in the Development of Sensation Seeking and Impulsivity During Adolescence : Further Evidence for a Dual Systems Model », Developmental Psychology, 47 (3) : 739.

Nallet-Buisson N., Makhlouf D., 2010, " Les psychologues au service de la sécurité routière ", Le Journal des psychologues, 279 : 62-67. 
Paire-Ficout L., Borgetto J., 2012, « Le code de la route reste peu accessible à un grand nombre de personnes sourdes ", Les Cahiers de l'audition, 25 (5) : 7-12.

Zuckerman M., 1979, Sensation Seeking, Hoboken, John Wiley \& Sons. 